\title{
Liquid Phase Deposition of Silica on the Hexagonally Close-Packed Monolayer of Silica Spheres
}

\author{
Seo Young Yoon, Sung-Eun Choi, and Jin Seok Lee \\ Department of Chemistry, Sookmyung Women's University, Seoul 140-742, Republic of Korea \\ Correspondence should be addressed to Jin Seok Lee; jinslee@sookmyung.ac.kr
}

Received 19 July 2013; Accepted 12 September 2013

Academic Editor: Guangyu Zhao

Copyright (c) 2013 Seo Young Yoon et al. This is an open access article distributed under the Creative Commons Attribution License, which permits unrestricted use, distribution, and reproduction in any medium, provided the original work is properly cited.

\begin{abstract}
Liquid phase deposition is a method used for the nonelectrochemical production of polycrystalline ceramic films at low temperatures, most commonly silicon dioxide films. Herein, we report that silica spheres are organized in a hexagonal close-packed array using a patterned substrate. On this monolayer of silica spheres, we could fabricate new nanostructures in which deposition and etching compete through a modified LPD reaction. In the early stage, silica spheres began to undergo etching, and then, silica bridges between the silica spheres appeared by the local deposition reaction. Finally, the silica spheres and bridges disappeared completely. We propose the mechanism for the formation of nanostructure.
\end{abstract}

\section{Introduction}

Silicon dioxide $\left(\mathrm{SiO}_{2}\right)$ films are widely useful in various fields. These films act as interlayer dielectrics and gate oxides in transistors and in the fabrication of integrated circuits and ultralarge-scale integration (ULSI) technologies [1-7]. In addition, $\mathrm{SiO}_{2}$ films play an important role in semiconductor devices as electrical insulators preventing the motion of carriers from [8] and could be universally applicable as one of the chief components of optical antireflection coatings and liquid crystal display (LCD) substrates as a mask to prevent alkali ions from diffusing to the ITO films [9]. Many techniques to produce $\mathrm{SiO}_{2}$ films have been researched, such as thermal oxidation, chemical vapor deposition (CVD), and sputtering $[7,9,10]$. However, these techniques have several drawbacks, mainly the need for specific equipments such as vacuum systems or glove boxes as well as expensive and sensitive organometallic precursors [11]. Furthermore, some techniques require a high reaction temperature of around several hundred degrees centigrade.

Recently, among various techniques, the liquid phase deposition (LPD) has been focused on as a useful method for the deposition of oxide films at low temperatures in aqueous solutions. LPD can overcome the abovementioned drawbacks of conventional methods because it does not require the use of vacuum systems and sensitive reagents. LPD progresses easily at room temperature, demands low production costs, and has minimal environmental impact. LPD is especially useful in coating not only flat substrates but also nonplanar substrates [11].

Up until now, research regarding LPD has investigated the formation and kinetics of films on planar substrates. In 1998, Nagayama et al. reported that $\mathrm{SiO}_{2}$ films could be deposited on glass in an $\mathrm{H}_{2} \mathrm{SiF}_{6}$ solution supersaturated with silica, after $\mathrm{H}_{3} \mathrm{BO}_{3}$ was added to the $\mathrm{H}_{2} \mathrm{SiF}_{6}$ solution which was saturated with $\mathrm{SiO}_{2}$ [12]. Whitsitt and Barron found that $\mathrm{SiO}_{2}$ films could be formed on substrates in an $\mathrm{H}_{2} \mathrm{SiF}_{6}$ solution supersaturated with $\mathrm{SiO}_{2}$ by dissolving aluminum instead of $\mathrm{H}_{3} \mathrm{BO}_{3}$ [11]. Further, Tsukuma et al. researched other silicic acid solutions having the unique ability to deposit films on a substrate and described the processing and properties of the silica film with some organic groups, deposited in the liquid phase [13]. However, most of the LPD research has been focused on flat substrates, and research on nanostructured substrates remains to be investigated. Kim and Roh fabricated isolated nanostructure by using the selective LPD on silicon substrates with grooves formed through etching [14]. Whitsitt and Barron researched different morphology of silica particles by LPD method with different surfactant [15]. Here, we investigated LPD on nanostructured surfaces 


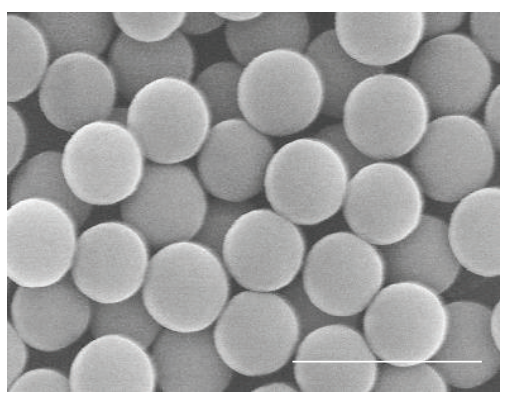

(a)

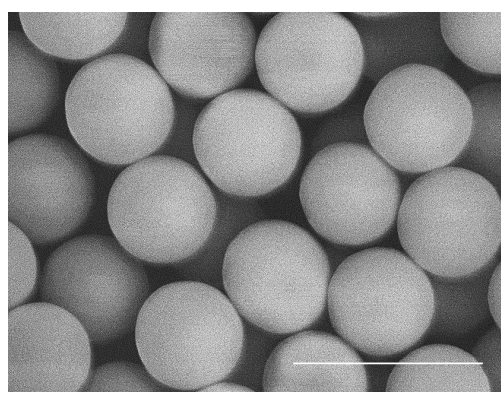

(b)

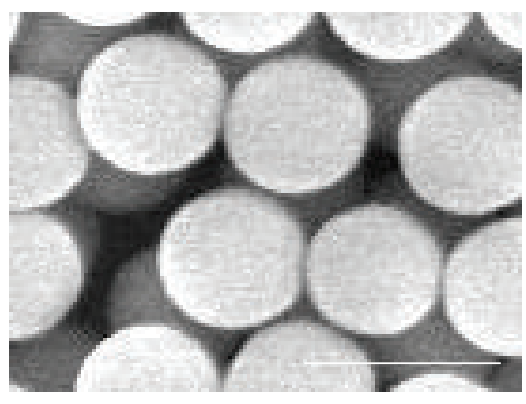

(c)

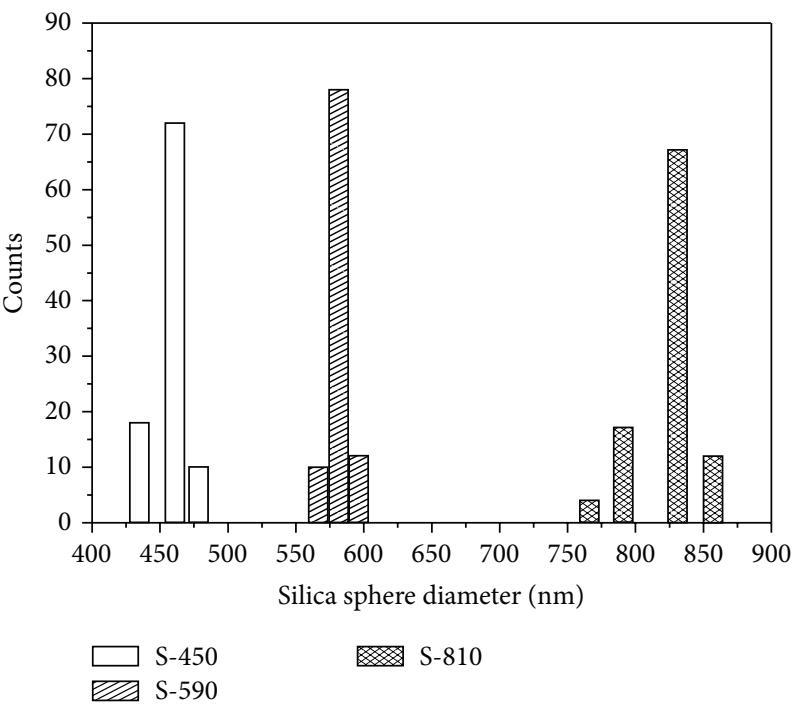

(d)

FIGURE 1: SEM images of silica spheres synthesized by the Stöber method. The average diameter of the silica spheres is $450 \mathrm{~nm}$ (a), $590 \mathrm{~nm}$ (b), and $810 \mathrm{~nm}$ (c), denoted by S-450, S-590, and S-810, respectively. The distribution of the diameters of silica spheres is shown in (d). Scale bars are $1 \mu \mathrm{m}$.

formed by the manual-assembled building blocks, which are different from previous research. For more effective production of oxide films, it is possible to either increase the concentration of water or decrease the concentration of hydrofluoric acid based on Le Chatelier's principle. To decrease the concentration of hydrofluoric acid, boric acid or aluminum metals are commonly used to function as fluoride $\left(\mathrm{F}^{-}\right)$scavengers [10]. Boric acid or aluminum metals could be used to produce more silicon dioxide; however, the use of these supplementary additives affects the film properties because other materials might be doped into $\mathrm{SiO}_{2}$ film and increase the impurity level. Consequently, the quality and insulating properties of the $\mathrm{SiO}_{2}$ film are degraded.

In this study, we assembled silica spheres with hexagonally close-packing by using a patterned substrate. Then, we performed a modified LPD reaction on the nanostructure, which meant the silica sphere array, without additives but by changing the saturation of hydrofluorosilicic acid $\left(\mathrm{H}_{2} \mathrm{SiF}_{6}\right)$. As a result, we fabricated a new nanostructure showing local deposition and etching reaction at the same time and proposed the mechanism of formation of the new nanostructure.

\section{Experiments}

2.1. Synthesis of Silica Spheres. Silica spheres were synthesized using the Stöber method, which involves the hydrolysis of tetraethyl orthosilicate (TEOS) in ethanol in the presence of ammonia $\left(\mathrm{NH}_{3}\right)$ as a catalyst $[16,17]$. By controlling the concentration of reactants, we changed the size of the silica spheres. Ammonia and ethanol adjusted to the proper concentrations were placed in a glass flask and stirred. After stabilizing the solution, TEOS was added and stirred to induce hydrolysis and condensation process. Finally, the silica suspension was centrifuged and washed by repeated redispersion in pure ethanol several times.

2.2. Assembly of Silica Spheres. The silicon wafer with a $300 \mathrm{~nm}$ thick $\mathrm{SiO}_{2}$ layer was coated with poly(methyl methacrylate) (PMMA) C2 by spin coating. The patterned Si substrates were immersed in piranha solution for $30 \mathrm{~min}$ and washed with deionized (DI) water. To prepare the poly(dimethylsiloxane) (PDMS) stamp, PDMS was poured onto the cleaned, patterned Si substrates and baked at $70^{\circ} \mathrm{C}$ for a few hours. Then, a small amount of the silica spheres 
powder was placed on the PDMS stamp and rubbed repeatedly in the same direction using a PDMS slab. After rubbing, the randomly aggregated upper layers of silica spheres were removed from the bottom layer with hexagonally closepacking (HCP) by using a fresh sticky PDMS slab for a few seconds on top of the silica sphere array and subsequently removing the PDMS slab. Finally, this monolayer of the HCP silica spheres on the PDMS stamp was transferred to the precoated silicon wafer with a $300 \mathrm{~nm}$ thick $\mathrm{SiO}_{2}$ layer with PMMA [18].

2.3. Liquid Phase Deposition of Nanostructure. As mentioned in the previous section, HCP silica spheres were heated at $200^{\circ} \mathrm{C}$ for $2 \mathrm{~min}$ to soften PMMA, which immobilized the silica spheres on PMMA layer. On the other hand, the liquid phase deposition solution was prepared as follows. First, $110 \mathrm{~mL}$ of hydrofluorosilicic acid $\left(\mathrm{H}_{2} \mathrm{SiF}_{6}, 35 \%\right)$ and $2 \mathrm{~g}$ of fumed silica $\left(\mathrm{SiO}_{2}\right)$ powder were mixed and stirred at $400 \mathrm{rpm}$ overnight. After stirring, this solution was filtered using a vacuum filtration, and DI water was added to the filtered solution at a ratio of $1: 2$ to allow for supersaturation with silicic acid. Immediately after preparing the solution, the samples were immersed in the solution for various times from 5 to $30 \mathrm{~min}$. After the reaction, the sample was rinsed with DI water and dried with nitrogen blowing.

\section{Results and Discussion}

We synthesized silica spheres with various sizes by controlling the concentrations of the reactants (Figure 1). Among them, S-590 was the most uniform. Narrow size distribution is an important factor in forming a uniform nanostructure consisting of silica spheres with a low density of defects using patterned substrates. We used the silica spheres with a diameter of around $600 \mathrm{~nm}$. The assembly process of silica spheres using patterned substrates is described in Figure 2. We fabricated a patterned Si substrate with hexagonal arrays of spherical protrusions. PDMS was poured and hardened on this patterned Si substrate, and then, a patterned PDMS stamp with hexagonal array of wells, contrary to the patterned Si substrate, was created (see the Supplementary Material for SEM images of patterned substrate available online at http://dx.doi.org/10.1155/2013/510524). After rubbing the silica spheres on the PDMS stamp, the monolayer of silica spheres was transferred onto the precoated silicon wafer with a $300 \mathrm{~nm}$ thick $\mathrm{SiO}_{2}$ layer with PMMA. One advantage of this method is the utilization of silica sphere powder rather than a dispersion solution of silica spheres. When the LangmuirBlodgett assembly method is used, a diluted dispersion solution of silica spheres that can suspend and spread along the interface between air and water is required [19, 20]. As the silica sphere array dries after the Langmuir-Blodgett assembly, water evaporates from them. This evaporation may influence the assembly of the silica spheres, which may crack because of particle shrinkage, but eventually, grain formation occurs. Although it is possible to rub silica sphere powder on glass, this method may lead to crack formation, as in the case of the Langmuir-Blodgett assembly. However, when the silica

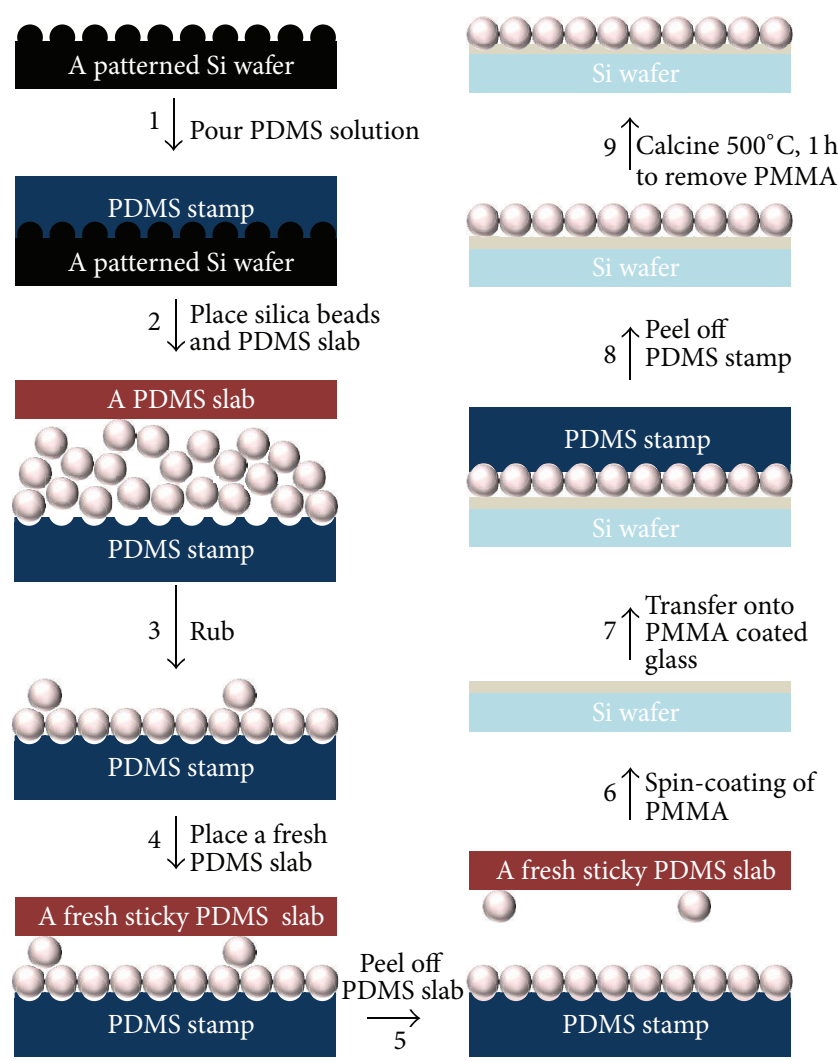

FIGURE 2: Schematic diagram of the general procedure to prepare perfect hexagonally close-packed arrays of silica spheres.

sphere powder is rubbed on the patterned Si wafer substrate, a perfect hexagonally close-packed assembly is formed over a very large area. Thus, this process is highly rapid and simple.

We conducted the LPD of silica on the hexagonally close-packed monolayer of silica spheres. The general LPD reaction, hydrofluorosilicic acid supersaturated with silicon dioxide is used to make the LPD solution. In this study, we experimented with LPD solutions consisting of unsaturated hydrofluorosilicic acid with $2 \mathrm{~g}$ of fumed silica powder, as the source of silicon dioxide. When supersaturated hydrofluorosilicic acid is filtered, undissolved silica substances are commonly sieved from the solution. However, our solution was not supersaturated because of insufficient silicon dioxide, and hence, there were no filtered substances. The experiment progressed using this LPD solution, and the results according to time are shown in Figure 3. In the general LPD reaction, silicon dioxide is homogeneously precipitated onto the substrate; however, we obtained surprising outcomes. Before the LPD reaction $(0 \mathrm{~min})$, the silica spheres were hexagonally close-packed (Figure 3(a)). After the LPD reaction for $5 \mathrm{~min}$, the silica sphere size was reduced. It should be emphasized that at this point, short and fine bridges between silica spheres started appearing (Figure 3(b)). The average length of the bridges and the standard deviation were $32.59 \mathrm{~nm}$ and $6.32 \mathrm{~nm}$, respectively. After another $5 \mathrm{~min}$, the silica spheres were continuously reduced, and the bridges became more elongated to $108.18 \mathrm{~nm} \pm 14.82 \mathrm{~nm}$ (Figure 3(c)). The 


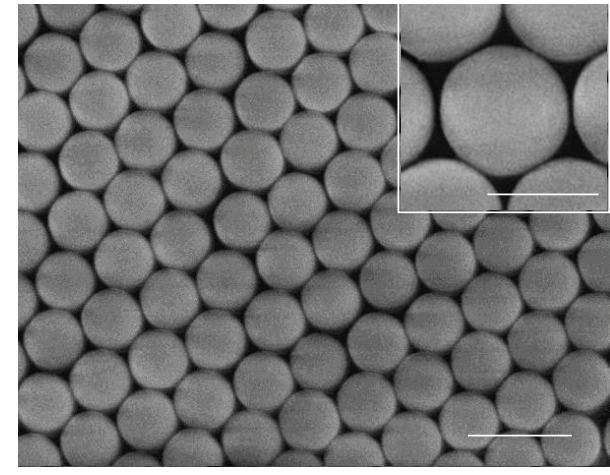

(a)

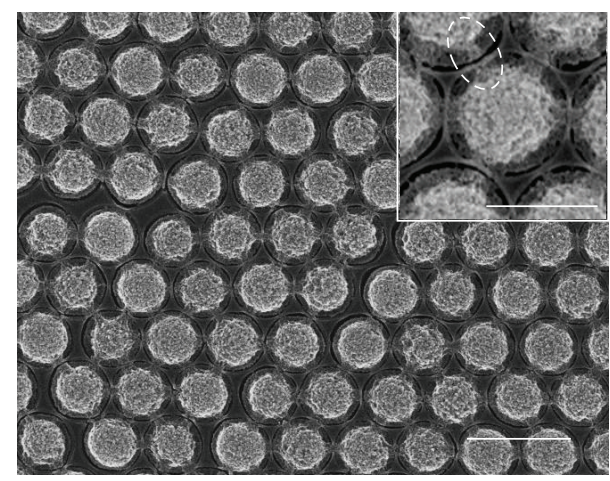

(c)

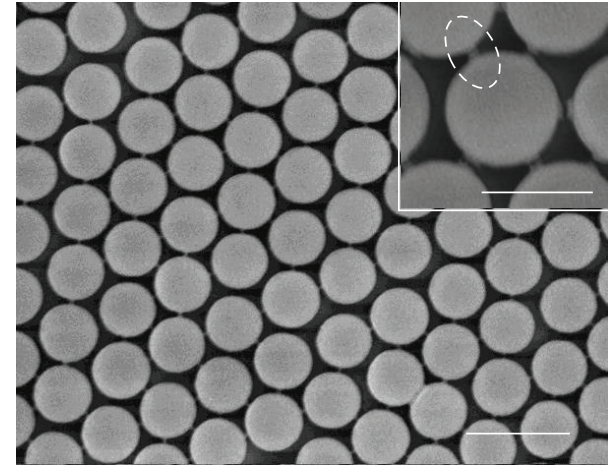

(b)

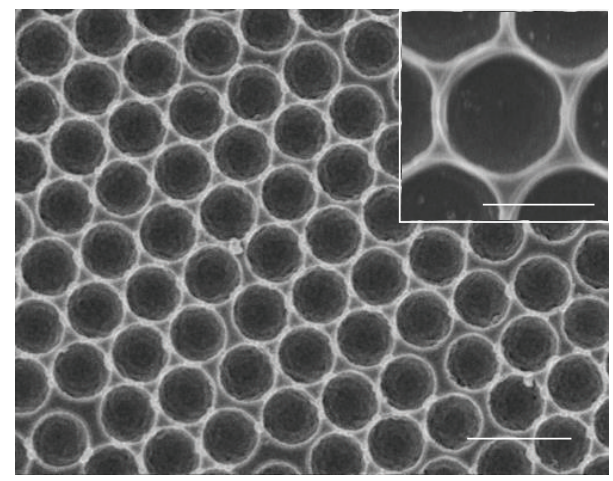

(d)

Figure 3: SEM images of liquid phase deposition according to the deposition time: (a) $0 \mathrm{~min}$, (b) $5 \mathrm{~min}$, (c) $10 \mathrm{~min}$, and (d) $30 \mathrm{~min}$. The dashed circle represents the bridge between silica spheres. Scale bars are $1 \mu \mathrm{m}$, and inset scale bars are $500 \mathrm{~nm}$.

surface of the silica spheres and the bridges after $10 \mathrm{~min}$ were different from those observed after $5 \mathrm{~min}$. Commonly, bridge formation is observed in both assemblies, which is the result of deposition. However, in Figure 3(c), the silica spheres seem to be very rough, which is an evidence of the etching reaction. Therefore, both reactions, that is, deposition and etching, occur simultaneously in one solution and compete with each other. After the LPD reaction in $30 \mathrm{~min}$, all the silica spheres and bridges were etched and eventually disappeared. Only a trace of the embedded silica spheres was discovered (Figure 3(d)). We reported a similar nanostructure in our previous work using the same processes: first, isotropic etching of silica spheres, formation of a spherebridge networks (SB-NWs), and eventual disappearance of the silica spheres and SB-NWs structure [21].

The changes in silica sphere size during the LPD reaction with time are shown in Figure 4(a). Once the reaction commenced, the silica spheres were etched and their size showed a consistent decrease. During the etching reaction, the silica spheres size decreased, and the bridge length between the silica spheres increased. Finally, the bridges disappeared after 30 min because of perfect etching. In our previous work, while the etching reaction was progressed, the etching rate of the silica sphere array decreased because the amount of hydrofluoric acid decreased. On the other hand, while the current LPD reaction progressed, silicon dioxides were formed in hydrofluorosilicic acid from the silica spheres by etching themselves. In accordance with this reaction, a large amount of hydrofluoric acid was produced because of the reaction between silicon dioxide and hydrofluorosilicic acid. For this reason, the reaction rate increased, in contrast to our previous work, and was probed throughout the slope of the graph. The slope tended to increase with time. Energy dispersive X-ray spectrometry (EDS) analysis of the silica spheres and networks are shown in Figures 4(b) and 4(c). The EDS spectra measured at the centers of the silica spheres and bridges confirmed the existence of $\mathrm{Si}$ and $\mathrm{O}$. The $\mathrm{Si} / \mathrm{O}$ atomic ratio in the silica sphere was estimated to be $1: 1.17$, which is quite similar to that of the bridge $(1: 1.20)$ and close to the stoichiometric composition of $\mathrm{SiO}_{2}$. Thus, this analysis confirms that the bridges are composed of $\mathrm{SiO}_{2}$ in a ratio essentially equal to that in the case of the silica spheres.

In our previous work, the sphere-bridge network structure was formed by etching using a neutral solution of 1:30 (v/v) mixture of 49\% $\mathrm{HF}$ acid and $40 \% \mathrm{NH}_{4} \mathrm{~F}$, and a mechanism for the formation of SB-NW structures was proposed. This work demonstrates an increased ammonium hexafluorosilicate (AHFS) concentration in the formed droplets at the interstitial sites of three adjacent silica spheres, and it indicates where the three materials, that is, AHFS, silica sphere, and aqueous silicic acid, are in contact, that is, the bridges formed between the silica spheres through the precipitation of silicic acids. As opposed to our previous work, in the present case, we used an LPD solution 


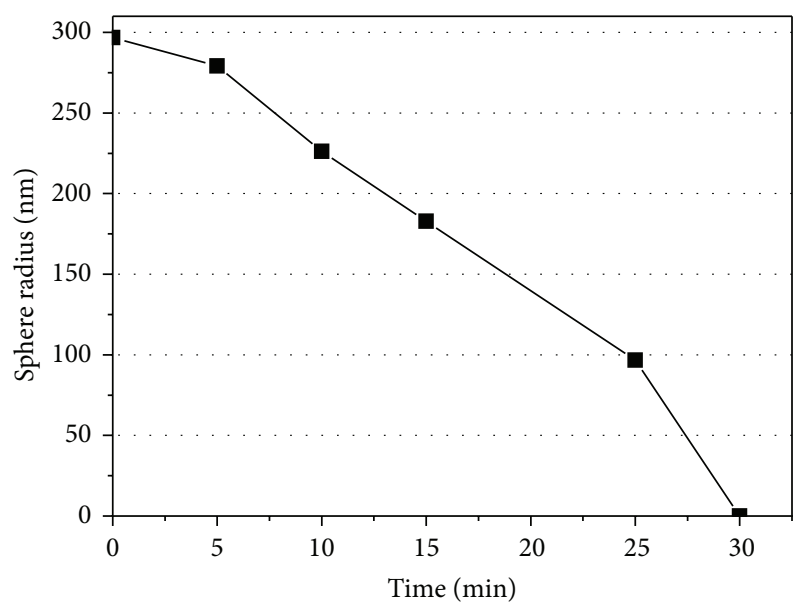

(a)

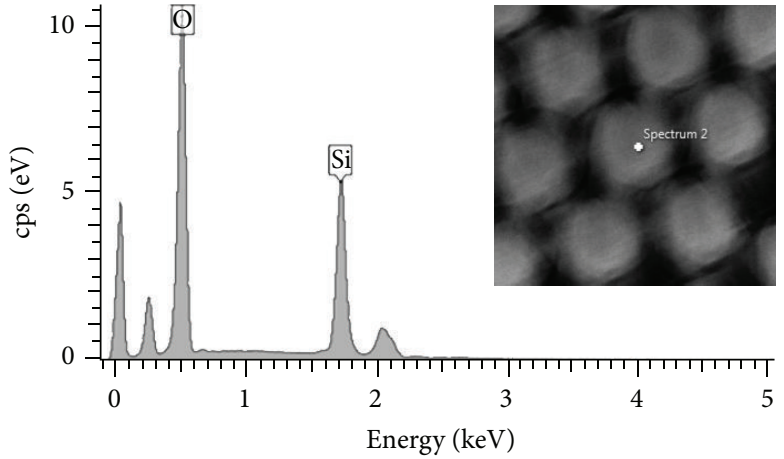

(b)

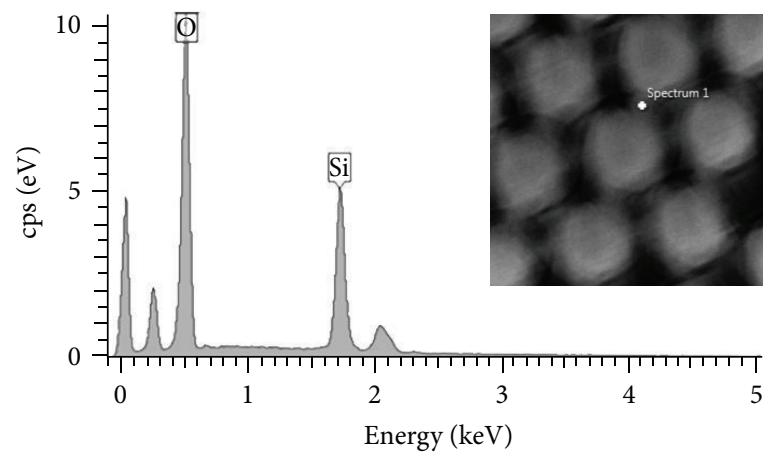

(c)

FIGURE 4: (a) Graph of the reaction rate of liquid phase deposition with respect to time. EDS analysis of the center of a silica sphere (b) and a bridge (c).

containing $2 \mathrm{~g}$ of fumed silica instead of a near neutral solution. In our LPD solution, the following reaction occurred [1]:

$$
\begin{aligned}
& \mathrm{H}_{2} \mathrm{SiF}_{6}+\mathrm{SiO}_{2} \longleftrightarrow 3\left[\mathrm{SiF}_{6} \mathrm{SiF}_{4}\right]^{2-}+2 \mathrm{H}_{2} \mathrm{O}+6 \mathrm{H}^{+} \\
& {\left[\mathrm{SiF}_{6} \mathrm{SiF}_{4}\right]^{2-}+2 \mathrm{H}_{2} \mathrm{O} \longleftrightarrow\left[\mathrm{SiF}_{4}(\mathrm{OH})_{2}\right]^{2-}+2 \mathrm{HF}}
\end{aligned}
$$

In these reactions, hydrofluoric acid could start etching the silica spheres, which were composed of silicon dioxide; accordingly, the size of the spheres was reduced. Meanwhile, silicic acid was produced at the same time as the etching reaction, as per the following reactions $[1,21]$ :

$$
\begin{gathered}
\mathrm{SiO}_{2}+6 \mathrm{HF} \longleftrightarrow \mathrm{H}_{2} \mathrm{SiF}_{6}+2 \mathrm{H}_{2} \mathrm{O} \\
\mathrm{H}_{2} \mathrm{SiF}_{6}+4 \mathrm{H}_{2} \mathrm{O} \longleftrightarrow \mathrm{Si}(\mathrm{OH})_{4}+6 \mathrm{HF} \\
{\left[\mathrm{SiF}_{4}(\mathrm{OH})_{2}\right]^{2-}+2 \mathrm{H}^{+} \longleftrightarrow \mathrm{SiF}_{4}+2 \mathrm{H}_{2} \mathrm{O}} \\
3 \mathrm{SiF}_{4}+4 \mathrm{H}_{2} \mathrm{O} \longleftrightarrow 2 \mathrm{SiF}_{6}{ }^{2-}+\mathrm{Si}(\mathrm{OH})_{4}+4 \mathrm{H}^{+}
\end{gathered}
$$

The concentration of silicic acid was much higher at the point of contact between the silica spheres than at any other site. At that contact point, therefore, polymerization of silicic acid could progress, leading to the formation of bridges between the silica spheres. In other words, the formation of bridges indicates a deposition reaction in the local region which the silica spheres contacted. Concurrently, the etching reaction occurs in the region of uncontacted silica spheres. Therefore, two reactions, deposition and etching, competed with each other. After lapse of time, according to the abovementioned process, the concentration of hydrofluoric acid increased, and hence, the etching reaction became more predominant. After the silica sphere size decreased and the bridges between silica spheres elongated, the bridges were also etched. Thus, all the silica spheres and bridges were etched and eventually disappeared. This final etching result is explained by (1) the equilibrium reaction of silicic acid and silicon dioxide and (2) the change in the reaction rate according to the acidic conditions. First, the equilibrium reaction of silicic acid and silicon dioxide occurs as follows:

$$
\mathrm{SiO}_{2}(\mathrm{~s})+2 \mathrm{H}_{2} \mathrm{O} \longleftrightarrow \mathrm{Si}(\mathrm{OH})_{4}(\mathrm{aq})
$$

In accordance with this reaction, the equilibrium constant is dependent on the concentration of silicic acid. While the LPD reaction progressed, the amount of silicic acids was insufficient. Therefore, the abovementioned reaction accelerated toward the formation of silicic acid and silicon dioxide, consistently supplied by the self-etching of the 


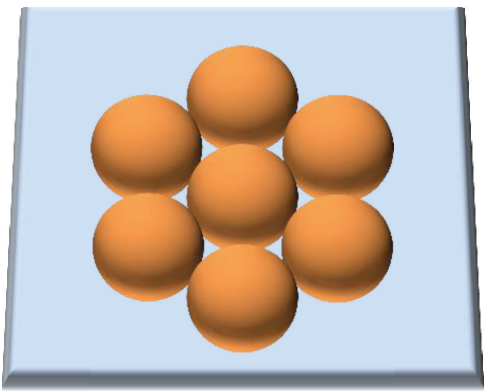

(a)

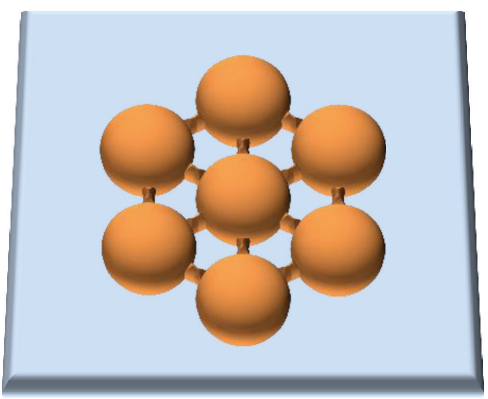

(d)

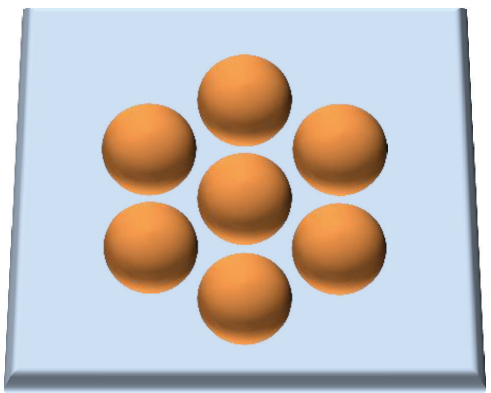

(b)

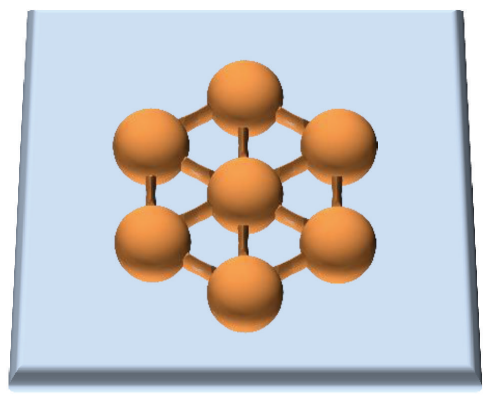

(e)

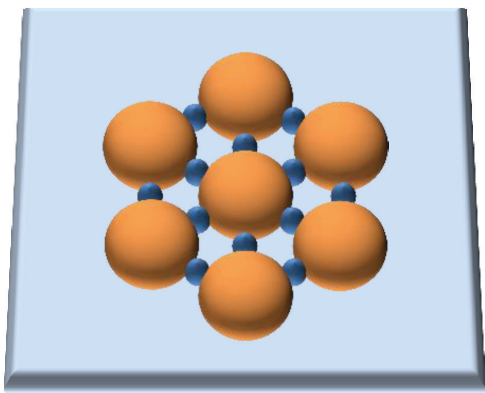

(c)

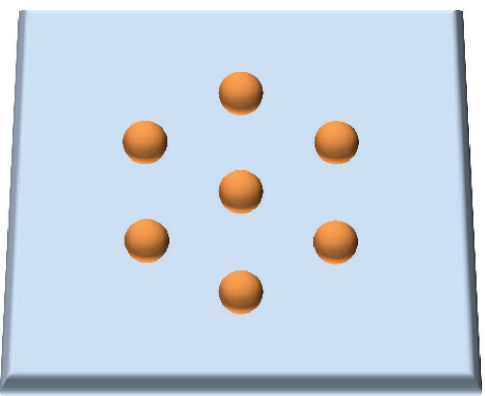

(f)

FIGURE 5: Schematic illustration depicting the mechanism of formation of nanostructure. (a) Hexagonally close-packed spheres in an aqueous solution. (b) Decrease in the size of silica spheres through homogeneous etching. (c) High concentration of silicic acid (blue) between the silica spheres. (d) Deposition of silica bridges between silica spheres. (e) Reduction of the nanostructure size due to etching. (f) Disappearance of the bridges.

silica spheres. Moreover, in the conditions under $\mathrm{pH} 7$, the rate of the condensation reaction rapidly decreases, but the rate of hydrolysis sharply increases [22]. Because this reaction progresses in acidic solutions, the hydrolysis reaction occurs more easily than the condensation reaction does. Through this mechanism, we can fabricate nanostructures that have not been traditionally obtained by general methods such as synthesis and growth. Figure 5 depicts in brief the mechanism for the formation of the nanostructure.

\section{Conclusions}

In this work, we fabricated an array of perfect hexagonally close-packed silica spheres over a large area of a patterned substrate. Then, we experimented with a modified LPD reaction on this nanostructured substrate and demonstrated a new nanostructure that certified simultaneous deposition and etching, that is, between these two processes; then, we proposed the mechanism for the formation of this nanostructure. This nanostructure could be useful in the biointerface field as a substrate that influences biological systems such as cell and bacteria. Because biological behaviors are dependent on the external environment, the unique surface topography of the nanostructure could trigger new biological responses such as cellular adhesion, development, differentiation, proliferation, and so on.

\section{Acknowledgment}

This work was supported by the SRC Research Center for Women's Diseases of Sookmyung Women's University (2010).

\section{References}

[1] S. E. Babayan, J. Y. Jeong, A. Schütze et al., "Deposition of silicon dioxide films with a non-equilibrium atmospheric-pressure plasma jet," Plasma Sources Science and Technology, vol. 10, no. 4, pp. 573-578, 2001.

[2] M. F. Ceiler Jr., P. A. Kohl, and S. A. Bidstrup, "Plasma-enhanced chemical vapor deposition of silicon dioxide deposited at low temperatures," Journal of the Electrochemical Society, vol. 142, no. 6, pp. 2067-2071, 1995.

[3] K. Ikeda, S. Nakayama, and M. Maeda, "Characteristics of silicon dioxide films on patterned substrates prepared by atmospheric-pressure chemical vapor deposition using tetraethoxysilane and ozone," Journal of the Electrochemical Society, vol. 143, no. 5, pp. 1715-1718, 1996.

[4] K. Murase, "Dielectric constant of silicon dioxide deposited by atmospheric-pressure chemical vapor deposition using tetraethylorthosilicate and ozone," Japanese Journal of Applied Physics, vol. 33, no. 3, pp. 1385-1389, 1994.

[5] W. J. Patrick, G. C. Schwartz, J. D. Chapple-Sokol, R. Carruthers, and K. Olsen, "Plasma-enhanced chemical vapor deposition of silicon dioxide films using tetraethoxysilane and oxygen: characterization and properties of films," Journal of the Electrochemical Society, vol. 139, no. 9, pp. 2604-2613, 1992. 
[6] S. K. Ray, C. K. Maiti, S. K. Lahiri, and N. B. Chakrabarti, "TEOS-based PECVD of silicon dioxide for VLSI applications," Advanced Materials for Optics and Electronics, vol. 6, no. 2, pp. 73-82, 1996.

[7] K. S. Kim and Y. Roh, "Silicon dioxide deposited by using liquid phase deposition at room temperature for nanometer-scaled isolation technology," Journal of the Korean Physical Society, vol. 51, no. 3, pp. 1191-1194, 2007.

[8] A. I. Kingon, J.-P. Maria, and S. K. Streiffer, "Alternative dielectrics to silicon dioxide for memory and logic devices," Nature, vol. 406, no. 6799, pp. 1032-1038, 2000.

[9] A. Hishinuma, T. Goda, M. Kitaoka, S. Hayashi, and H. Kawahara, "Formation of silicon dioxide films in acidic solutions," Applied Surface Science, vol. 48-49, pp. 405-408, 1991.

[10] A. V. Valiulis and P. Silickas, "Liquid phase deposition methods monitoring techniques influence for solid substrates and thin metal oxide films properties," Journal of Achievements in Materials and Manufacturing Engineering, vol. 24, pp. 188-192, 2007.

[11] E. A. Whitsitt and A. R. Barron, Liquid phase deposition of silica: thin films, colloids and fullerenes [Ph.D. thesis], 2004.

[12] H. Nagayama, H. Honda, and H. Kawahara, "New process for silica coating solid-state science and technology," Journal of the Electrochemical Society, vol. 135, no. 8, pp. 2013-2016, 1988.

[13] K. Tsukuma, T. Akiyama, N. Yamada, and H. Imai, "Liquid phase deposition of a film of silica with an organic functional group," Journal of Non-Crystalline Solids, vol. 231, no. 1-2, pp. 161-168, 1998.

[14] K. S. Kim and Y. Roh, "Silicon dioxide deposited by using liquid phase deposition at room temperature for nanometer-scaled isolation technology," Journal of the Korean Physical Society, vol. 51, no. 3, pp. 1191-1194, 2007.

[15] E. A. Whitsitt and A. R. Barron, "Effect of surfactant on particle morphology for liquid phase deposition of submicron silica," Journal of Colloid and Interface Science, vol. 287, no. 1, pp. 318325, 2005.

[16] W. Stöber, A. Fink, and E. Bohn, "Controlled growth of monodisperse silica spheres in the micron size range," Journal of Colloid And Interface Science, vol. 26, no. 1, pp. 62-69, 1968.

[17] K. Nozawa, H. Gailhanou, L. Raison et al., "Smart control of monodisperse stöber silica particles: effect of reactant addition rate on growth process," Langmuir, vol. 21, no. 4, pp. 1516-1523, 2005.

[18] N. N. Khanh and B. Y. Kyung, "Facile organization of colloidal particles into large, perfect one-and two-dimensional arrays by dry manual assembly on patterned substrates," Journal of the American Chemical Society, vol. 131, no. 40, pp. 14228-14230, 2009.

[19] S. Reculusa and S. Ravaine, "Synthesis of colloidal crystals of controllable thickness through the Langmuir-Blodgett technique," Chemistry of Materials, vol. 15, no. 2, pp. 598-605, 2003.

[20] J. Zhu, Z. Yu, G. F. Burkhart et al., "Optical absorption enhancement in amorphous silicon nanowire and nanocone arrays," Nano Letters, vol. 9, no. 1, pp. 279-282, 2009.

[21] J. Yi, H. S. Jang, J. S. Lee, and W. I. Park, "Bioinspired morphogenesis of highly intricate and symmetric silica nanostructures," Nano Letters, vol. 12, pp. 743-3748, 2012.

[22] C. J. Brinker and G. W. Scherer, Sol-Gel Science: the Physic and Chemistry of Sol-Gel Processing, Academic Press, San Diego, Calif, USA, 1990. 

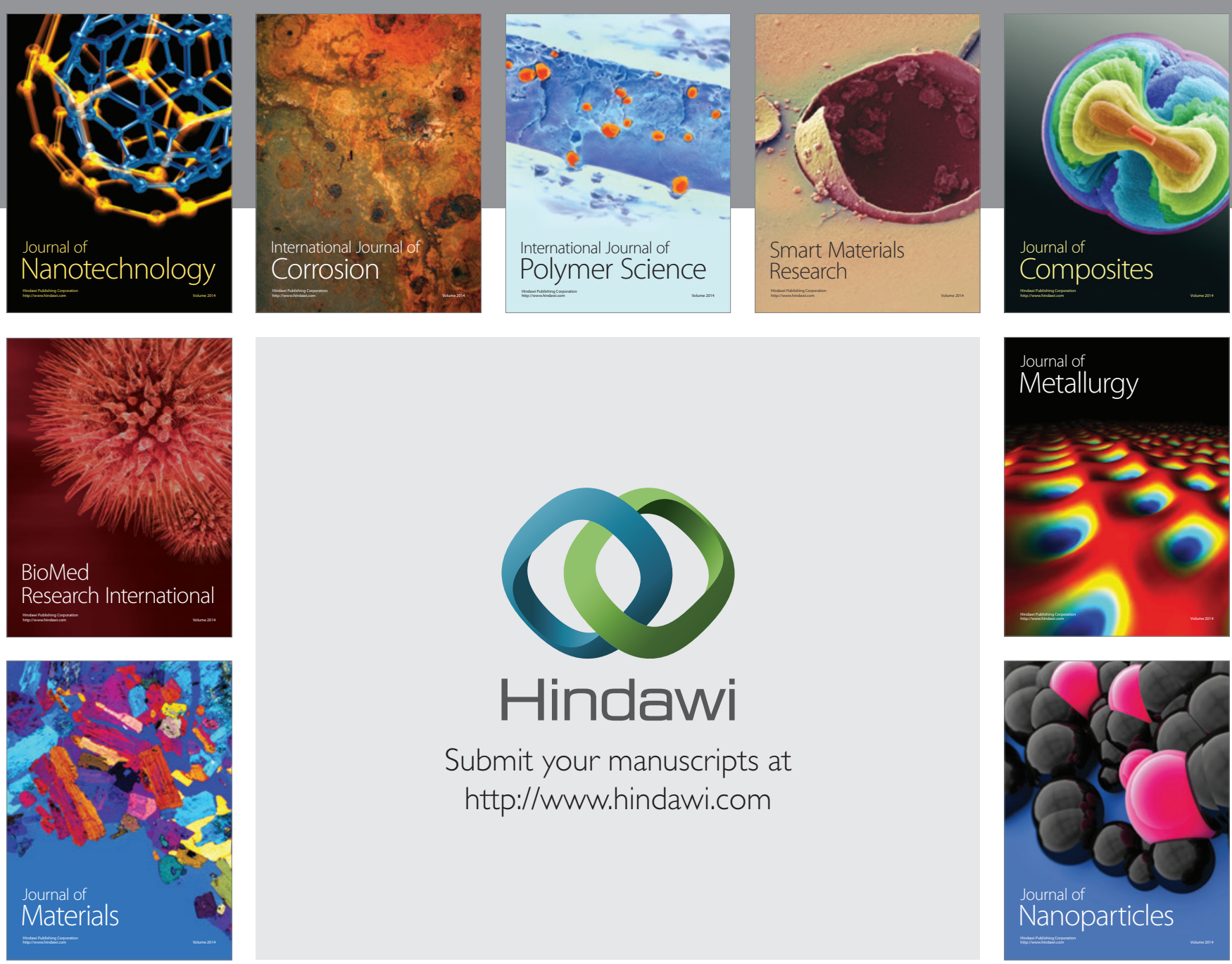

Submit your manuscripts at http://www.hindawi.com
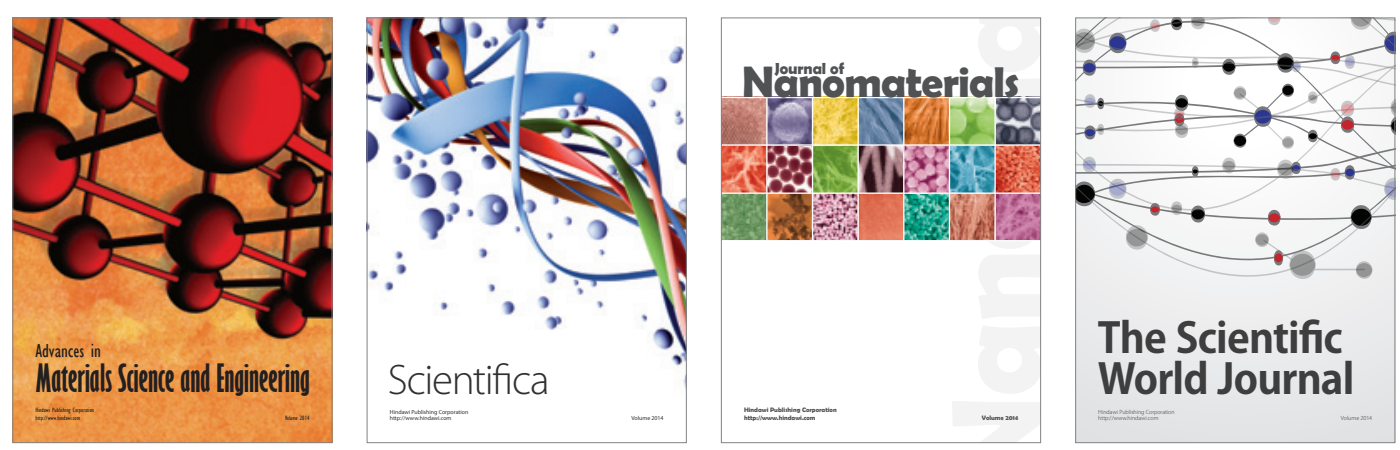

\section{The Scientific World Journal}
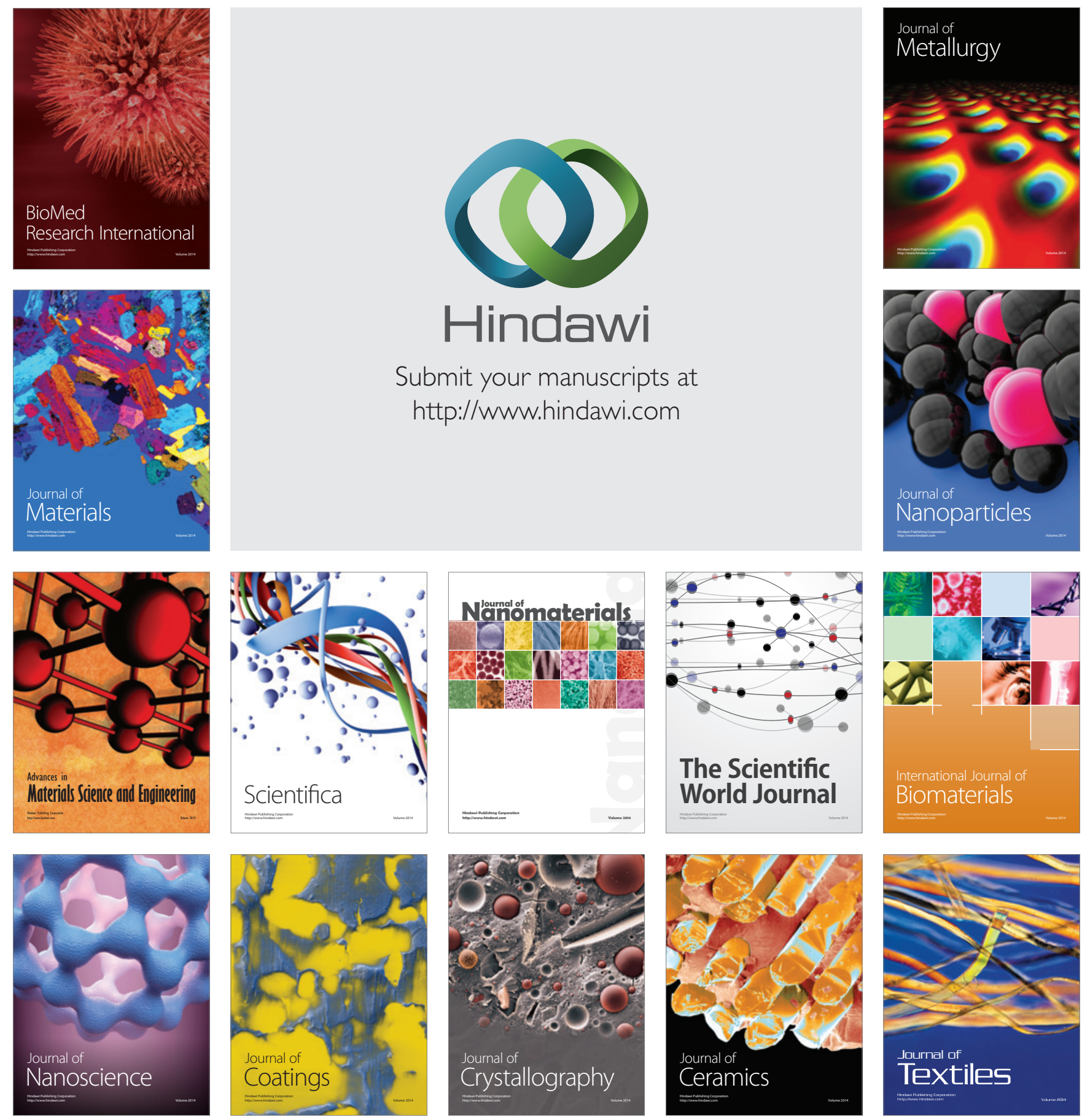